\title{
Subspace Predictive Control for Continuous-time Systems
}

\author{
Xiaosuo Luo \\ ${ }^{1}$ School of Automation, Chongqing University, Chongqing, China, 400044 \\ ${ }^{2}$ Chongqing College of Electronic Engineering, Chongqing, China, 401331 \\ lock86@126.com
}

\begin{abstract}
The paper presents a model predictive control method for continuous-time systems based on subspace identification. It's developed by reformulating the continuous-time systems using Laguerre filters to obtain the subspace prediction output. Then, the subspace predictors are derived by $Q R$ decomposition from input-output and Laguerre matrices. The subspace predictive controller is designed with the subspace predictors. The process control simulations of a fermentation bioreactor system show the effectiveness of the proposed method.
\end{abstract}

Keywords: Continuous-time systems, Model predictive control, Subspace identification, Fermentation bioreactor

\section{Introduction}

The industrial processes become more complex than before and many of them are continuous-time systems, such as continuous rolling process, the aircraft flight process and so on [1-2]. Because of the complexity of the continuous processes, the models are often difficult to obtain from the mechanism modeling, it is necessary to use the identification method. Generally, the continuous-time identification falls into two distinguish categories: The indirect approach and direct approach [3]. The indirect approach basically view the situation at two points: First by using a non-parametric model like impulse response, step response or frequency response function. Second step is to estimate continuous time parameters from the estimated discrete time model. The drawbacks of the indirect approach are that the sampling time is difficult to select and zero pole conversion is not consistent. In contrast, the direct approach often approximates the derivative operator that is associated with input and output signal using a filter to identify the continuous-time models directly. Compared to the indirect approach, the direct approach is more superior and turns into focus of study [4]. Subspace identification is one of system identification algorithms for state-space modeling. The control workers may relieve completely from the tedious mechanism modeling and the accurate statespace model can be obtained when there is enough process input-output data [5-6]. Model predictive control(MPC) has been attractive for decades in control theory field. It has become more established as the one of the choices for the control architecture in the industry, especially with the improvement of computational capabilities of processors [78]. By combining the merits of subspace identification and MPC, subspace predictive control(SPC) was formed [9-10]. It's very useful to design the direct controller of continuous-time systems which can create the favorable condition for the project implementation of SPC.

The subspace identification of continuous-time systems has been studied in a number of contributions. In [11] an approach for system identification of continuous-time stochastic state space models from random input-output continuous data was presented. The approach is based on the introduction of random distribution theory in describing (higher) time derivatives of stochastic processes, and the input-output algebraic 
relationship is derived which is treated in the time-domain. Wu et al. [12] solved the continuous-time identification for errors-in-variables based on the linear filter method and principal component analysis. Bergamasco and Lovera [13] dealt with the problem of continuous-time model identification and presented two subspace-based algorithms capable of dealing with data generated by systems operating in closed loop. These methods only solve the identification problem. In this paper, we extend continuous-time subspace identification to design the predictive controller using subspace predictors.

In this brief, different from previous works, a novel subspace predictive control method is developed for continuous-time systems. The continuous-time system is first transformed into the Laguerre form to obtain the subspace prediction output. The subspace predictors are derived from input-output and Laguerre matrices, then they are used to design the predictive controller. Superior control performance is obtained than adopting discrete-time subspace identification method for continuous-time systems.

The paper is arranged as follows. Section 2 provides the subspace prediction output for continuous-time system. Section 3 gives the SPC method for continuous-time system. The control method is simulated in Section 4. Section 5 ends with the conclusions.

\section{Subspace Prediction Output for Continuous-Time Systems}

Consider a continuous-time state-space system described by stochastic form

$$
\begin{aligned}
& x(t)=A x(t)+B u(t)+w(t) \\
& y(t)=C x(t)+D u(t)+v(t)
\end{aligned}
$$

where $u(t) \in \mathrm{i}^{\prime}, y(t) \in \mathrm{i}^{m}$, and $x(t) \in \mathrm{i}^{n}$ are input, output, and state vectors respectively. $(A, B, C, D)$ are system matrices of appropriate dimensions. $w(t) \in \mathrm{R}^{n}$ and $v(t) \in \mathrm{R}^{m}$ are zero-mean white Gaussian sequences with covariance matrix:

$$
\mathrm{E}\left[\left(\begin{array}{l}
w(t) \\
v(t)
\end{array}\right)\left(\begin{array}{ll}
w(t)^{\mathrm{T}} & \left.v(t)^{\mathrm{T}}\right)
\end{array}\right]=\left[\begin{array}{ll}
Q & S \\
S^{\mathrm{T}} & R
\end{array}\right] \delta_{i j}\right.
$$

where $\delta_{i j}$ is Kronecker delta.

The $i$-th continuous-time Laguerre filter is given by

$$
L_{i}(s)=\sqrt{2 p} \frac{(s-a)^{i}}{(s+a)^{i+1}}
$$

where $a>0$ is the scaling factor to ensure that the filters are stable. Define a $w$-operator that corresponds to the all-pass Laguerre filter which has the form

$$
w(s)=\frac{s-a}{s+a}
$$

Through multiplication, the system can be transformed as follows [14],

where

$$
\begin{aligned}
& {[w x](t)=A_{w} x(t)+B_{w}\left[l_{0} u\right](t)+\left[l_{0} w_{w}\right](t)+K_{1} x_{0} l_{0}(t)} \\
& {\left[l_{0} y\right](t)=C_{w} x(t)+D_{w}\left[l_{0} u\right](t)+\left[l_{0} v_{w}\right](t)+K_{2} x_{0} l_{0}(t)}
\end{aligned}
$$




$$
\begin{aligned}
& A_{w}=(A+a I)^{-1}(A-a I), \\
& B_{w}=(A+a I)^{-1} B, \\
& C_{w}=2 a C(A+a I)^{-1}, \\
& D_{w}=D-C(A+a I)^{-1} B, \\
& K_{1}=(A+a I)^{-1}, \\
& K_{2}=C(A+a I)^{-1}, \\
& w_{w}(t)=(A+a I)^{-1} w(t), \\
& v_{w}(t)=v(t)-C(A+a I)^{-1} w(t),
\end{aligned}
$$

and $x_{0}$ is the initial state of the original continuous-time system.

The subspace prediction output of the continuous-time system can be derived by recursive substitution of Eqs. (6)-(7):

$$
Y_{i, j}(t)=\Gamma_{j} x(t)+H_{j} U_{i, j}(t)+H_{j}^{s} W_{i, j}(t)+V_{i, j}(t)+F_{j} \Psi_{i, j}(t)
$$

where

$$
\left.Y_{i, j}(t)=\left[\begin{array}{c}
{\left[l_{i} y\right](t)} \\
{\left[l_{i+1} y\right](t)} \\
\vdots \\
{\left[l_{i+j-1} y\right](t)}
\end{array}\right] \quad, \quad \Gamma_{j}=\left\{\begin{array}{c}
C_{w} \\
C_{w} A_{w} \\
\vdots \\
C_{w} A_{w}^{j-1}
\end{array}\right] \quad, \quad H_{j}=\begin{array}{ccccc}
D_{w} & 0 & \cdots & 0 \\
C_{w} B_{w} & D_{w} & \ddots & \vdots \\
\vdots & \ddots & \ddots & 0 \\
C_{w} A_{w}^{j-2} B_{w} & \cdots & C_{w} B_{w} & D_{w}
\end{array}\right],
$$$$
U_{i, j}(t)=\left[\begin{array}{c}
{\left[l_{i} u\right](t)} \\
{\left[l_{i+1} u\right](t)} \\
\vdots \\
{\left[l_{i+j-1} u\right](t)}
\end{array}\right] \quad, \quad H_{j}^{s}=\left[\begin{array}{cccc}
0 & 0 & \cdots & 0 \\
C_{w} & 0 & \ddots & \vdots \\
\vdots & \ddots & \ddots & 0 \\
C_{w} A_{w}^{j-2} & \cdots & C_{w} & 0
\end{array}\right] \quad, \quad W_{i, j}(t)=\left[\begin{array}{c}
{\left[l_{i} w_{w}\right](t)} \\
{\left[l_{i+1} w_{w}\right](t)} \\
\vdots \\
{[} \\
{\left[l_{i+j-1} w_{w}\right](t)}
\end{array}\right],
$$$$
V_{i, j}(t)=\left[\begin{array}{c}
{\left[l_{i} v_{w}\right](t)} \\
{\left[l_{i+1} v_{w}\right](t)} \\
\vdots \\
{\left[l_{i+j-1} v_{w}\right](t)}
\end{array}\right], \Psi_{i, j}(t)=\left\{\begin{array}{c}
l_{i}(t) \\
l_{i+1}(t) \\
\vdots \\
l_{i+j-1}(t)
\end{array}\right], F_{j}=\left\{\begin{array}{cccc}
K_{2} x_{0} & 0 & \cdots & 0 \\
C_{w} K_{1} x_{0} & K_{2} x_{0} & \ddots & \vdots \\
\vdots & \ddots & \ddots & 0 \\
C_{w} A_{w}^{j-2} K_{1} x_{0} & \cdots & C_{w} K_{1} x_{0} & K_{2} x_{0}
\end{array}\right] .
$$

\section{Subspace Predictive Control for Continuous-Time Systems}

Construct the following input-output and Laguerre matrices with $N$ samples through subspace prediction output:

$$
\left[\begin{array}{l}
\Psi_{p} \\
W_{p} \\
U_{f} \\
Y_{f}
\end{array}\left|=R^{\mathrm{T}} Q^{\mathrm{T}}=\left[\begin{array}{lcccc}
R_{11} & 0 & 0 & 0 & 7\left\lceil Q_{1}^{\mathrm{T}}\right. \\
R_{21} & R_{22} & 0 & 0 & \| \\
R_{31} & R_{32} & R_{33} & 0 & \| Q_{2}^{\mathrm{T}} \\
R_{41} & R_{42} & R_{43} & R_{44}
\end{array}\right\rfloor\right|\left[\begin{array}{l}
\mathrm{T} \\
Q_{4}^{\mathrm{T}}
\end{array}\right]\right.
$$

where the subscripts $p$ and $f$ represent the 'past' and 'future' time, $R$ is a low triangular matrix, and $Q$ is an orthogonal matrix. And 


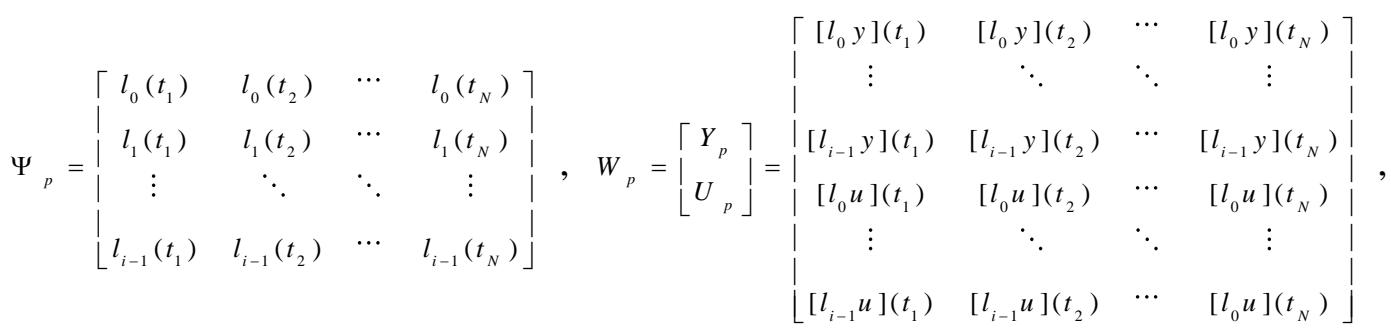

$$
\begin{aligned}
& U_{f}=\left[\begin{array}{cccc}
{\left[l_{i} u\right]\left(t_{1}\right)} & {\left[l_{i} u\right]\left(t_{2}\right)} & \cdots & {\left[l_{i} u\right]\left(t_{N}\right)} \\
{\left[l_{i+1} u\right]\left(t_{1}\right)} & {\left[l_{i+1} u\right]\left(t_{2}\right)} & \cdots & {\left[l_{i+1} u\right]\left(t_{N}\right)} \\
\vdots & \ddots & \ddots & \vdots \\
{\left[l_{i+j-1} u\right]\left(t_{1}\right)} & {\left[l_{i+j-1} u\right]\left(t_{2}\right)} & \cdots & {\left[l_{i+j-1} u\right]\left(t_{N}\right)}
\end{array}\right], \\
& Y_{f}=\left\{\begin{array}{cccc}
{\left[l_{i} y\right]\left(t_{1}\right)} & {\left[l_{i} y\right]\left(t_{2}\right)} & \cdots & {\left[l_{i} y\right]\left(t_{N}\right)} \\
{\left[l_{i+1} y\right]\left(t_{1}\right)} & {\left[l_{i+1} y\right]\left(t_{2}\right)} & \cdots & {\left[l_{i+1} y\right]\left(t_{N}\right)} \\
\vdots & \ddots & \ddots & \vdots \\
{\left[l_{i+j-1} y\right]\left(t_{1}\right)} & {\left[l_{i+j-1} y\right]\left(t_{2}\right)} & \cdots & {\left[l_{i+j-1} y\right]\left(t_{N}\right)}
\end{array}\right] .
\end{aligned}
$$

The optimal prediction $Y_{f}$ can be found from the orthogonal projection of the row space of $Y_{f}$ onto the row space of the matrix $\left[\begin{array}{ll}\Psi_{p} \\ W_{p} \\ W_{p} \\ U_{f}\end{array}\right]$ :

$$
\hat{Y}_{f}=Y_{f} /\left[\begin{array}{c}
\Psi_{p} \\
W_{p} \\
U_{f}
\end{array}\right]=L_{w} W_{p}+L_{u} U_{f}
$$

where $L_{w}$ is the subspace predictor that corresponds to the past input-output data and $L_{u}$ is the subspace predictor that corresponds to the future input data.

Implement the orthogonal projection, we can get

$$
\left[\begin{array}{ll}
L_{w} & L_{u}
\end{array}\right]=\left[\begin{array}{lll}
R_{41} & R_{42} & R_{43}
\end{array}\right]\left[\begin{array}{lll}
R_{21} & R_{22} & 0 \\
R_{31} & R_{32} & R_{33}
\end{array}\right]^{\dagger}
$$

where superscript $\uparrow$ represents the Moore-Penrose pseudo-inverse. Next, the subspace predictors are used to design predictive controller.

A typical form of cost function in MPC is given as follows:

$$
\begin{aligned}
J & =\sum_{k=1}^{N_{2}}\left(r_{t+k}-\hat{y}_{t+k \mid t}\right)^{2}+\sum_{j=1}^{N_{u}} \lambda\left(\Delta u_{t+j-1}\right)^{2} \\
& =\left(r_{f}-\hat{y}_{f}\right)^{\mathrm{T}}\left(r_{f}-\hat{y}_{f}\right)+\Delta u_{f}^{\mathrm{T}}(\lambda I) \Delta u_{f}
\end{aligned}
$$

where $N_{2}$ and $N_{u}$ are the prediction and control horizon respectively, $r_{t+k}$ is the reference setpoint signal at the current time $t+k, \lambda$ is the weighting on the control effort. The vector of the optimal prediction of the future outputs can be expressed in terms of the future inputs and current states as

$$
\hat{y}_{f}=F y_{t}+L_{w}^{\circ}\left(1: N_{2} m,:\right) \Delta w_{p}+S_{N_{2}, N_{u}} \Delta u_{f}
$$

where $\hat{y}_{f}=\left[\begin{array}{lll}\hat{y}_{t+1} & \cdots & \hat{y}_{t+N_{2}}\end{array}\right]^{\mathrm{T}}, F=\left[\begin{array}{lll}I_{m} & \cdots & I_{m}\end{array}\right]^{\mathrm{T}}$, 


$$
\begin{gathered}
S_{N_{2}, N_{u}}=L_{u}\left(1: N_{2} m, 1: N_{u} l\right)\left[\begin{array}{cccc}
I_{l} & 0 & \cdots & 0 \\
I_{l} & I_{l} & \cdots & 0 \\
\vdots & \vdots & \ddots & \vdots \\
I_{l} & I_{l} & \cdots & I_{l}
\end{array}\right] \cdot L_{w}^{\circ} \text { is constructed from } L_{w} \text { as } \\
L_{w}^{\circ}(m(k-1)+1: m k,:)=\sum_{i=1}^{k} L_{w}(m(i-1)+1: m i,:)
\end{gathered}
$$

where $1 \leq k \leq N_{2}$. Substituting the optimal prediction of the future outputs in Eq. (14) into the cost function in Eq. (13), differentiate it with respect to $\Delta u_{f}$ and control sequence can be obtained:

$$
\Delta u_{f}=\left(S_{N_{2}, N_{u}}^{\mathrm{T}} S_{N_{2}, N_{u}}+\lambda I\right)^{-1} S_{N_{2}, N_{u}}^{\mathrm{T}}\left(r_{f}-F y_{t}-L_{w}^{\circ}\left(1: N_{2} m,:\right) \Delta w_{p}\right)
$$

At each time instance, only the first element of $\Delta u_{f}$ is used for calculating the control input. Therefore the control input $u_{t}$ is drawn as

$$
u_{t}=u_{t-1}+\Delta u_{t}
$$

At the next time step, measuring the new input-output data and the new control input will be calculated using above optimization.

\section{Simulation Example}

The fermentation bioreactor system is a typical continuous-time system. Among the bioreactors, the alcohol fermentation is one of the most important biochemical processes, the system diagram shown in Figure 1 [15].

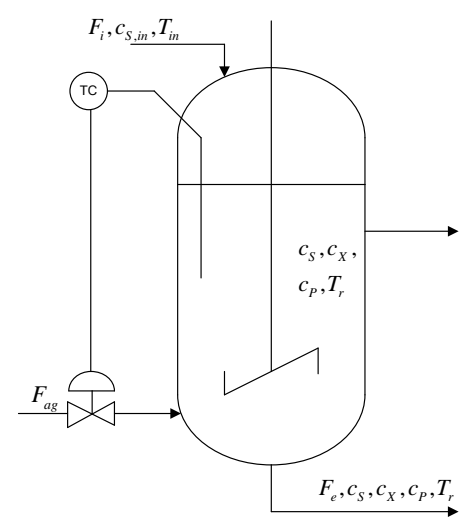

Figure 1. Fermentation Bioreactor System Diagram

Maintaining the balance of the volume, mass, and energy, the nonlinear differential equations of this system can be described as follows: 


$$
\left\{\begin{array}{l}
\left\{\begin{array}{l}
\frac{d V}{d t}=F_{i}-F_{e}, \\
\frac{d c_{X}}{d t}=\mu_{X} c_{X} \frac{c_{S}}{K_{S}+c_{S}} e^{-K_{P} c_{P}}-\frac{F_{e}}{V} c_{X}, \\
\frac{d c_{P}}{d t}=\mu_{P} c_{X} \frac{c_{S}}{K_{S 1}+c_{S}} e^{-K_{P} c_{P}}-\frac{F_{e}}{V} c_{P}, \\
\frac{d c_{S}}{d t}=-\frac{1}{R_{S X}} \mu_{X} c_{X} \frac{c_{S}}{K_{S}+c_{S}} e^{-K_{P} c_{P}}-\frac{1}{R_{S P}} \mu_{P} c_{X} \frac{c_{S}}{K_{S 1}+c_{S}} e^{-K_{P 1} c_{P}}+\frac{F_{i}}{V} c_{S, \text { in }}-\frac{F_{e}}{V} c_{S}, \\
\frac{d c_{\mathrm{O}_{2}}}{d t}=\left(k_{l} a\right)\left(c_{\mathrm{O}_{2}}^{*}-c_{\mathrm{O}_{2}}\right)-r_{\mathrm{O}_{2}}, \\
\frac{d T_{r}}{d t}=\frac{F_{i}}{V}\left(T_{i n}+273\right)-\frac{F_{e}}{V}\left(T_{r}+273\right)+\frac{r_{\mathrm{O}_{2}} \Delta H_{r}}{32 \rho_{r} C_{\text {heat }, r}}+\frac{K_{T} A_{T}\left(T_{r}-T_{a g}\right)}{V \rho_{r} C_{\text {heat }, r}}, \\
\frac{d T_{a g}}{d t}=\frac{F_{a g}}{V_{j}}\left(T_{i n, a g}-T_{a g}\right)+\frac{K_{T} A_{T}\left(T_{r}-T_{a g}\right)}{V_{j} \rho_{a g} C_{\text {heat }, a g}} .
\end{array}\right.
\end{array}\right.
$$

the system inputs are cooling water flow $F_{a g}$, feed concentration $c_{s, i n}$, feed flow $F_{i}$, product feed $F_{e}$, and feed temperature $T_{i n}$. The outputs are reactor volume $V$, cell concentration $c_{x}$, ethanol concentration $c_{P}$, glucose concentration $c_{S}$, oxygen concentration $c_{\mathrm{O}_{2}}$, reactor temperature $T_{r}$, and cooling water temperature $T_{a g}$. The physical process constants and operating point can be seen in [15].

Keeping the feed flow $F_{i}$ equal to the product feed $F_{e}$ and the reactor volume $V$ unchanged. When the cooling water flow $F_{a g}$ changes from $18 l / h$ to $9 l / h$, the open-loop response of the system is shown in Figure 2. It's showed strong nonlinearity in system and it's not easy to control the system to obtain the satisfying performance.
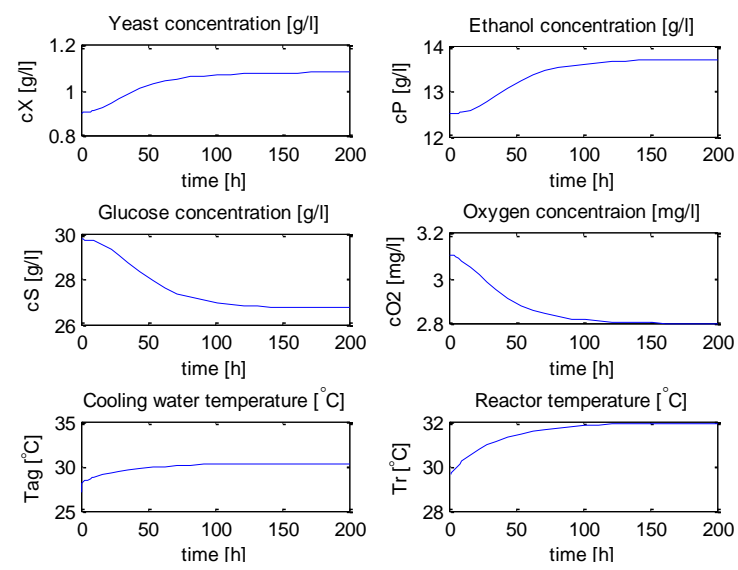

Figure 2. The Open-Loop Response of the System

The product quality depends on the reactor temperature $T_{r}$, so the target is set for $T_{r}$ tracking the reference signal in the system. Firstly, we used the continuous-time subspace method(CSM) in this paper and the discrete-time subspace method(DSM) in [10]. The input $F_{a g}$ was chosen to be a Gaussian random signal shifting by $\pm 30 \%$. The length of samples and sampling time were set to 400 and $1 \mathrm{~h}$ respectively, totally 150 samples were used to verify the identification accuracy. The 
comparisons in Figure 3 show that the response of the identified model and process output using CSM and DSM, where 'rf' is process output.

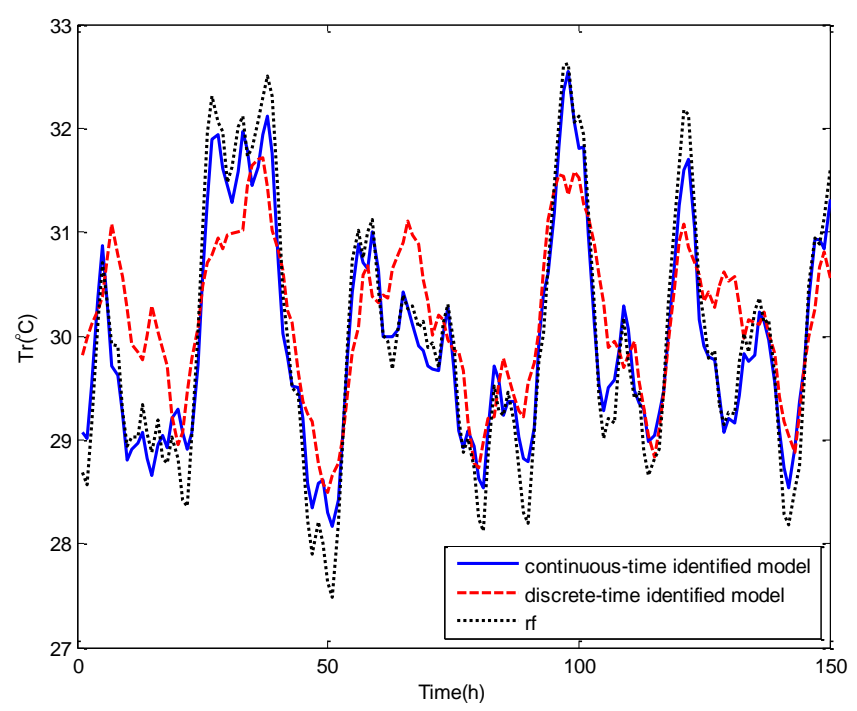

Figure 3. The Response of the Identified Model and Process Output

To test the cross validation in Figure 3, a form of prediction error is defined as:

$$
\xi=\sqrt{\frac{\sum_{i=1}^{N}\left(y_{i}-y_{i}^{p}\right)^{2}}{\sum_{i=1}^{N}\left(y_{i}\right)^{2}}}
$$

where $y_{i}$ and $y_{i}^{p}$ are the values at instant $i$ of process and model output respectively. The prediction error on the validation data set can be seen in Table 1. The cross validation results indicate that the continuous-time identified model is more accurate than discrete-time identified model.

Table 1. The Prediction Errors of Continuous-Time and Discrete-Time Identified Model

\begin{tabular}{|c|c|c|}
\hline Identified method & Continuous-time & Discrete-time \\
\hline Prediction error $\xi$ & 0.2709 & 0.5236 \\
\hline
\end{tabular}

Then, the identified models were used to design the predictive controllers. The parameters of continuous-time subspace predictive control(CSPC) method were tuned as follows. The prediction and control horizon $N_{2}=N_{u}=3, \lambda=5, N=200$, $t=1 \mathrm{~h}$. The initial value of $T_{r}$ was $30.76^{\circ} \mathrm{C}$, and the target is set to $T_{r}=32^{\circ} \mathrm{C}$. For comparison, the discrete-time subspace predictive control(DSPC) method in [10] and a PID controller were selected as competitors to compare the tracking capability. Figure 4 depicts the tracking comparison of these three controllers, where 'Ref' is set point value. It can be depicted that CSPC shows the favorable control performance and has a better tracking ability compared to DSPC and PID. 


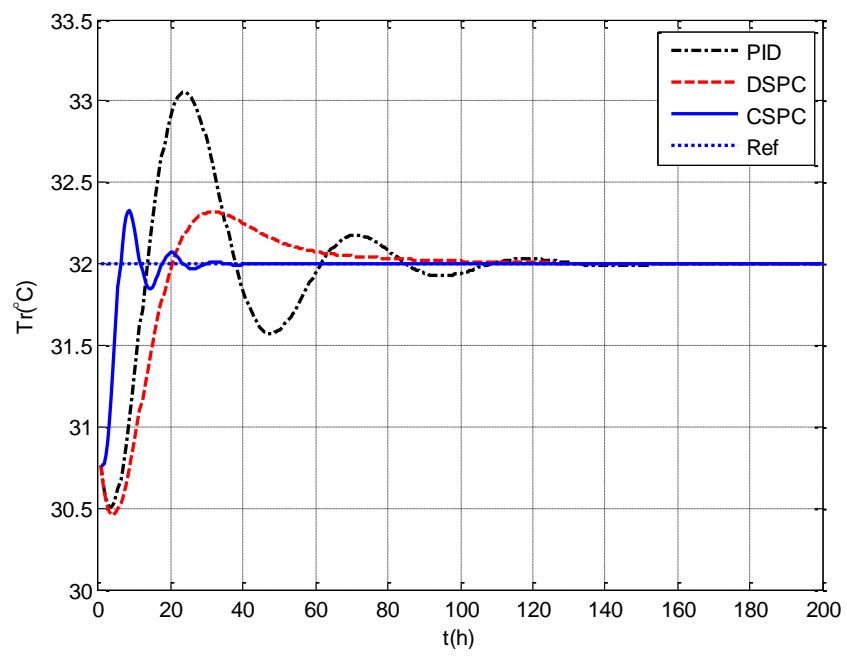

Figure 4. $T_{r}$ Tracking Performance

\section{Conclusions}

In this paper, the design of a subspace predictive controller for continuous-time systems has been addressed. The Laguerre filters are used to get the subspace prediction output. Then, the subspace predictors are obtained from input-output and Laguerre matrices, and the subspace predictive controller is designed using the subspace predictors. By simulation studies for the bioreactor example its performance has been proved to be efficient by comparing with other methods.

\section{Acknowledgements}

This work was supported in part by the Major State Basic Research Development Program 973 (No. 2012CB215202), the National Natural Science Foundation of China (No.61134001) and Key Laboratory of Dependable Service Computing in Cyber Physical Society (Chongqing University), Ministry of Education.

\section{References}

[1] H. Unbehauen and G.P. Rao, "A review of identification in continuous-time systems", Annual Reviews in Control, vol.22, (1998), pp.145-171.

[2] M. B. Tischler and R. K. Remple, "Aircraft and rotorcraft system identification", American Institute of Aeronautics and Astronautics, New York, (2006).

[3] H. Garnier and L. P. Wang, "Identification of continuous-time models from sampled data", Springer, Berlin, (2008).

[4] P. C. Young and H. Garnier, "Identification and estimation of continuous-time, data-based mechanistic (DBM) models for environmental systems", Environmental modelling \& software, vol.21, no.8, (2006), pp.1055-1072.

[5] W. Favoreel, B. D. Moor and P. V. Overschee, "Subspace state space system identification for industrial processes", Journal of Process Control, vol.10, no.2-3, (2000), pp.149-155.

[6] S. J. Qin, "An overview of subspace identification. Computers and Chemical Engineering”, vol.30, no.10-12, (2006), pp.1502-1513.

[7] K. S. Holkar and L. M. Waghmare, "An Overview of Model Predictive Control”, International Journal of Control and Automation, vol.3, no.4, (2010), pp.47-63.

[8] N. A. Wahab, R. Katebi, J. Balderud and M. F. Rahmat, "Data-driven adaptive model-based predictive control with application in wastewater systems", IET Control Theory and Applications, vol.5, no.6, (2011), pp.803-812.

[9] R. Hollouzi and M. Verhaegen, "Fault-Tolerant Subspace Predictive Control Applied to a Boeing 747 Model", Journal of Guidance, Control, and Dynamics, vol.31, no.4, (2008), pp.873-883.

[10] N. A. Mardi, "Data-driven subspace-based model predictive control". PhD thesis, RMIT University, August, (2010). 
[11] A. Ohsumi, K. Kameyama and K. I. Yamaguchi, "Subspace identification for continuous-time stochastic systems via distribution-based approach", Automatica, vol.38, no.1, (2002), pp.63-79.

[12] P. Wu, C. J. Yang and Z. H. Song, "Subspace identification for continuous-time errors-in-variables model from sampled data", Journal of Zhejiang University, Science-A, vol.10, no.8, (2009), pp.11771186.

[13] M. Bergamasco and M. Lovera, "Continuous-time predictor-based subspace identification using Laguerre filters”, IET Control Theory \& Applications, vol.5, no.7, (2011), pp.856-867.

[14] B. R. J. Haverkamp, "State space identification: theory and practice", PhD thesis, Delft University of Technology, (2001).

[15] Z. K. Nagy, "Model based control of a yeast fermentation bioreactor using optimally designed artificial neural networks", Chemical Engineering Journal, vol.127, no.1, (2007), pp.95-109.

\section{Author}

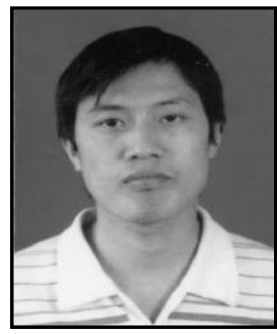

Xiaosuo Luo, he received his M.S. degree in School of Automation from Chongqing University, Chongqing, China, in 2011. He is currently a Ph.D. candidate at the School of Automation, Chongqing University. His research interests include subspace identification and model predictive control. 
International Journal of Control and Automation Vol. 8, No. 7 (2015) 